\title{
MEDICINA Y SEGURIDAD DEL TRABAJO HA SUPERADO LOS CRITERIOS DE SELECCIÓN PARA INTEGRARSE EN LA RED SCIELO (SCIENTIFIC ELECTRONIC LIBRARY ONLINE)
}

\author{
JORGE VEIGA DE CABO*
}

(*) Redactor Jefe Revista Medicina y Seguridad del Trabajo

La revista Medicina y Seguridad del Trabajo ha superado los criterios de selección para poder integrarse en la Red SciELO (Scientific Electronic Library Online), por lo que a partir del primer número de 2007, se podrá acceder al texto completo a la misma desde esta plataforma. Esto supone un logro importante, en la medida que pasa a formar parte del contenido de uno de los portales que goza de mayor visibilidad internacional, y uno de los de mayor acceso entre los países de habla hispana, contribuyendo así a su objetivo de expansión y ganando cotas de difusión, tanto nacional como internacional.
Por tanto, se podrá acceder al texto completo en formato electrónico a través de la página Web del Instituto de Salud Carlos III(http://www.isciii.es), entrando en la página de la Escuela Nacional de Medicina del Trabajo y directamente a través del espacio dedicado a la Revista de Medicina y Seguridad del Trabajo, o mediante el acceso a traves de la Red SciELO (http://www.sxcielo.org) o SciELOEspaña (http://scielo.isciii.es).

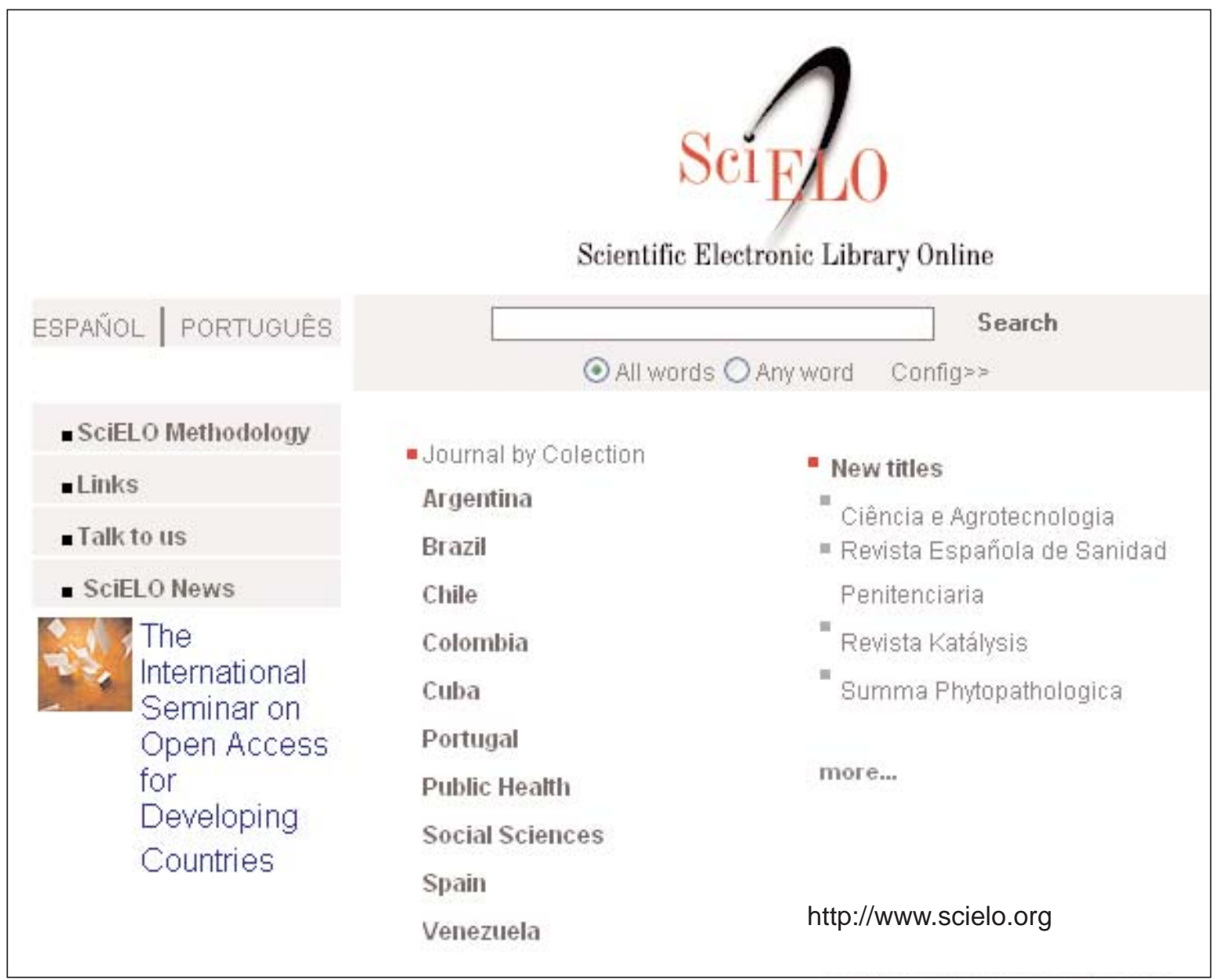




\section{¿QUÉ ES SCIELO?}

Scientific Electronic Library Online (SciELO) (http://www.scielo.org) está considerado como uno de las iniciativas Open Access más importantes de ámbito internacional.

Su objetivo es facilitar el acceso y la difusión de publicaciones científicas de calidad contrastada, editadas en países de Latinoamérica, España y Portugal. Para ello cuenta con criterios de selección previamente establecidos, entre los que se tienen en cuenta 44 items relacionados con calidad de edición, contenido y visibilidad.

La Red SciELO se inicia como proyecto piloto en 1997 en Sao Paulo (Brasil), liderado por el Centro Latinoamericano de Información y Documentación en Ciencias de la Salud (BIREME) de la Organización Panamericana de La Salud de la Organización Mundial de la Salud (OPSOMS)(http://www.paho.org).

Durante el transcurso del X Encuentro de la Escuela de Verano de Salud Pública (EVSP) celebrado en el Lazareto de Mahón (Menorca), del 20 al 24 de Septiembre de 1999, la Biblioteca Nacional de Ciencias de la Salud del Instituto de Salud Carlos III (BNCS/ISCIII), adquirió el compromiso de integración en la red SciELO, con el desarrollo de SciEloEspaña.

La Red SciELO, permite el acceso a texto completo a 400 títulos de revistas científicas de diferentes áreas temáticas, de las cuales, unas 160 se corresponden con publicaciones periódicas de ciencias de la salud de diferentes países de Latinoamérica, Portugal y España (Tabla 1).

Tabla I. Distribución por países de publicaciones periódicas accesibles desde la Red SciELO

\begin{tabular}{|c|c|c|}
\hline País & $\begin{array}{c}\text { Todas áreas } \\
\text { temáticas }\end{array}$ & $\begin{array}{c}\text { Ciencias de } \\
\text { la Salud }\end{array}$ \\
\hline Argentina & 28 & 5 \\
\hline Brasil & 178 & 65 \\
\hline Chile & 63 & 16 \\
\hline Colombia & 32 & 12 \\
\hline Cuba & 20 & 20 \\
\hline España & 31 & 31 \\
\hline Portugal & 15 & 3 \\
\hline Venezuela & 33 & 11 \\
\hline Total & $\mathbf{4 0 0}$ & $\mathbf{1 6 3}$ \\
\hline
\end{tabular}

El modelo SciELO integra diferentes componentes que permiten:

- Acceso al texto completo en formato electrónico (HTML y/o PDF), buscando por título de la revista, autor, palabras del título, materia, año de publicación y afiliación, de forma individual o combinando campos

- Acceder desde la bibliografía de un artículo a los registros bibliográficos de resúmenes y contenidos de bases de datos bibliográficas internacionales como Medline, PubMed Central y LILACS cuando éstos se encuentran referenciados en ellas, así como al texto completo, si el artículo ha sido editado anteriormente en SciELO.

- Obtener de forma directa la referencia de cómo citar el artículo mediante normas ISO (formato impreso y electrónico).

- Acceder a la Red internacional de investigadores SciENTI que facilita información complementaria sobre líneas de investigación y publicaciones de los autores de los artículos y

- Acceso al módulo de utilización, uso e impacto de las publicaciones periódicas integradas en la red SciELO.

Por último, la plataforma SciELO enlaza con otras plataformas de acceso electrónico como PubMed, WoS, DOAJ, OCLC, WordCat, LoC, LILACS, MedLine, IBECS y BioMed Central, entre otros.

\section{IMPACTO INTERNACIONAL DE SCIELO}

Un artículo que no se encuentra visible, accesible y disponible, sea por el procedimiento que sea, no se puede considerar integrado y con calado en la comunidad científica, objeto último de cualquier publicación.

Tradicionalmente, la visibilidad de las publicaciones se ha medido mediante el Factor de Impacto (FI), dato obtenido del análisis estadístico del número de veces que ha sido citada por el Institute for Scientific Information (ISI).

Desafortunadamente, este sistema de medición cuenta con notables desajustes y sesgos, ampliamente expuestos en la literatura científica, pero, ante la escasez de otros sistemas de medición alternativos o complementarios, el Factor de Impacto ha constituido y constituye, el principal sistema de medición de 
la visibilidad dentro de la cadena de producción científica. Además, el factor de impacto de una revista, no es representativo del factor de citación de un artículo editado en ella, como en ocasiones erróneamente se utiliza.

La aparición de la iniciativa Open Access ha contribuido a que aparezcan formas alternativas de medición de la visibilidad, que además, cada vez toman más fuerza en la era de la edición electrónica. Así tenemos, sistemas de medición de visibilidad como los Hits (número de veces que se accede al documento), Downloads (número de veces que se descarga un documento) y la Visibilidad (inclusión de una publicación como enlace en algún portal, plataforma o espacio Web).

En términos generales, la presencia de las publicaciones en soporte electrónico como complemento a la edición en papel, sobre todo mediante plataformas acreditadas Open Access, suponen uno de los sistemas más importantes para incrementar la visibilidad, y en consecuencia, hits, downloads y el mismo factor de impacto de las publicaciones.

Los resultados de análisis de unos 120.000 artículos del campo de la informática, realizado por Lawrence ${ }^{1}$, muestran una tendencia generalizada superior de incremento del impacto, cuando las publicaciones se encuentran complementadas con acceso libre a través de alguna plataforma Open Access, que cuando la edición se realiza únicamente en soporte papel.

Estudios similares han sido realizados por el propio $\mathrm{ISI}^{2}$, midiendo las variaciones y tendencias del factor de impacto de unas 200 publicaciones incluidas en algún sistema Open Access, respecto a las que utilizaban medios convencionales. De las 148 publicaciones monotorizadas y seguidas desde 2002, que entraron en el Journal Citation Report (JCR) hasta la finalización del estudio, encontraron benéficos en cuanto a que el índice de citación se producía mucho antes, es decir, una considerable mejora del índice de inmediatez. Otros estudios similares, mostraron como las pblicaciones incluídas en alguna plataforma Open Access, incrementaban entre el $32.2 \%$ y el $56.7 \%$ su impacto ${ }^{3}$.
Uno de los primero datos que permitieron relacionar la visibilidad alcanzada por la presencia de las revistas incluidas en SciELO con el incremento de impacto de las mismas, fueron los resultados de un estudio realizado por la Universidad de Oxford y publicado en la prestigiosa revista Nature en el año 2002. Los autores del artículo, seleccionaron cinco revistas indexadas por ISI durante más de cinco años y que se mantuvieron accesibles desde SciELO durante un periodo superior a dos años. Los factores de impacto de dichas revistas se habían incrementado en más del doble $(132.7 \% \mathrm{p}<0.02)$ desde su inclusión en SciELO ${ }^{4}$.

Otro ejemplo en la misma dirección, fue el estudio presentado por Lewis Joel Greene, director de la revista Brazilian Journal of Medical and Biological Research, publicación indizada en Medline y en ISI, durante la II Reunión Regional de la Red SciELO celebrada en Mérida, México (octubre 2002), y en el que se daba a conocer un dato igualmente indicativo de la difusión que pueden alcanzar los contenidos de SciELO, destacando el aumento del 100\% del número de citas y del factor de impacto en ISI de la revista desde su ingreso en SciELO.

Es difícil predecir la repercusión que pueda suponer para la revista de Medicina y Seguridad del Trabajo, su inclusión en la Red SciELO. En cualquier caso, podrán empezarse a notar a medio o largo plazo, más si tenemos en cuenta que los principales efectos en este sentido se comenzaron a notar a partir de los dos y cinco años, en revistas de excelencia e indizadas en ISI y JRC.

Lo que sí parece claro, es que a partir de este momento la revista adquiere su propio espacio en el escaparate internacional y entra en una dimensión diferente en lo que respecta a sus posibilidades de difusión y visibilidad universal. Además de los datos sobre factor de impacto que han sido presentados, que desde la consolidación de SciELO como Red internacional y su indización en Google, el índice de accesos se vio incrementado en valores cercanos al $80 \%$, recibiendo en la actualidad cerca de 2.5 millones de visitas mensuales de los más diferentes países del mundo, cifra que se encuentra en aumento ${ }^{5}$. 


\section{BIBLIOGRAFÍA}

1. Lawrence S. Free online availability sudstantiaally increases a paper's impact. Nature 2001; 411: 521-523.

2. Testa J, McVeigh ME. The impact of Open Access Journals. A citation study from Thomson ISI. Disponible en http://www.isinet.com/media/presenttrep/acropdf/impactoa-journals.pdf

3. Harnad S, Brody T. Comparing the impact of open access (OA) vs non-OA articles in the journals. D-Lib Magazine, Junio 2004. Disponible en: http://www.dlib.org/dlib/june04/harnad/06harnad.html
4. Alonso WJ, Fernández-Juricic E. Regional network raises profile of local journals [carta]. Nature, 2002; 415: 471-472.

5. Veiga de Cabo J. Scientific Electronic Library Online y Oncología. Oncología (Barc.) 2004: 27 (9); 13-18. Disponible en: http://wwwscielo.isciii.es/scielo. ph p ? script = sci_art text \& pid = S 0378 48352004000900001\&lng=es\&nrm=iso 